\title{
Successful Management of a Complex Case of Recurrent Sphenoid Sinus Mucocele with Fungal Sinusitis and Optic Nerve Compression
}

\author{
Shantanu Panja ${ }^{1}$, Mehak Agarwal ${ }^{2}$
}

\begin{abstract}
Background: Mucoceles occur most commonly in frontal sinus and rarely in sphenoid sinus. The common presentations in sphenoid sinus mucoceles are deep-seated headache and visual disturbance.

Case description: We present the case of a 58-year-old lady who came to us with headache, nose block, and reduced vision in the left eye since 2 years. She was diagnosed with an expansile lesion compressing the optic nerve. She underwent endoscopic drainage multiple times. The histopathological examination (HPE) was suggestive of aspergillus and she was treated with voriconazole. In spite of medical and surgical treatment, her symptoms were persistent. Eventually, she presented to our hospital with worsening of symptoms and deterioration of vision. Following MRI, we did drainage of mucocele with marsupialization. The clival bone was drilled out widening the entire face of sphenoid sinus. Fungal culture was suggestive of aspergillus and the patient was given voriconazole for 3 months. She has been on regular follow-up since then. Conclusion: In cases of recurrent sphenoid sinus mucoceles, complete marsupialization of the mucocele, drilling of bony barriers, adequate medical management, and diligent follow-up are necessary for the successful outcome.

Clinical significance: Recurrent mucoceles of the sphenoid sinus represent a difficult entity to treat and are ridden with complications. Not only do they require aggressive surgical management but also meticulous postoperative medical management and repeated endoscopic follow-ups. Keywords: Aspergillus, Marsupialization, Sphenoid mucocele.

Clinical Rhinology An International Journal (2020): 10.5005/jp-journals-10013-1366
\end{abstract}

\section{BACKGROUND}

Mucoceles of paranasal sinuses (PNS) are benign expansile cystic lesions that occur most commonly in frontal sinus and rarely in sphenoid sinus. The common presentations are deep-seated headache and visual disturbance. Although pathologically benign, they may involve vital structures like dura, pituitary gland, optic nerve, cavernous sinus, and internal carotid artery (ICA). Here, we would like to present a case of recurrent sphenoid sinus mucocele operated multiple times but presented to us with recurrent disease and associated complication.

\section{Case Description}

A 58-year-old lady presented with intermittent headache and nose block since 3 years and reduced vision in the left eye, which had progressively increased over 2 years.

Past history revealed that she had taken treatment and undergone surgery at a hospital outside for the above-said complaints. The details are as follows.

The MRI of orbit and brain (July 2016) showed an expansile lesion of sphenoid sinus, extending till the left optic foramen with optic nerve compression. Vision was $6 / 9$ in both eyes then, with loss of the temporal field of vision in the left.

The patient underwent endoscopic drainage of left sphenoid mucocele in July 2016. Histopathological examination (HPE) was suggestive of aspergillus. The patient had taken voriconazole orally for 4 months after surgery.

MRI PNS with contrast (Dec 2016) was suggestive of a sphenoid sinus lesion measuring $26.3 \times 33.3 \times 23.9 \mathrm{~mm}$ with the left parasellar
1,2Department of ENT and Head and Neck Surgery, Apollo Gleneagles Hospitals, Kolkata, West Bengal, India

Corresponding Author: Mehak Agarwal, Department of ENT and Head and Neck Surgery, Apollo Gleneagles Hospitals, Kolkata, West Bengal, India, Phone: +91 9599901755, e-mail: mehakagarwal07@gmail.com

How to cite this article: Panja S, Agarwal M. Successful Management of a Complex Case of Recurrent Sphenoid Sinus Mucocele with Fungal Sinusitis and Optic Nerve Compression. Clin Rhinol An Int J 2020;13(1):27-29.

Source of support: Nil

Conflict of interest: None

extension compressing the left ICA, superiorly compressing the pituitary gland, reaching up to left optic foramina. Repeat MRI (March 2017) showed no change from the previous imaging.

Subsequently, she underwent endoscopic drainage, synechia release, and septoplasty in March 2017 after which she was treated conservatively using antibiotics, anti-inflammatory medicines, nasal sprays, and decongestants.

Eventually, she presented to our hospital with worsening of symptoms. Her vision had deteriorated to $6 / 24$ in left with optic degeneration. MRI PNS and nasopharynx (plain and contrast) in Feb 2018 showed an expansile cystic lesion in sphenoid sinus more on the left side extending into posterior ethmoids, into left parasellar region, encasing left cavernous sinus and cavernous segment of left ICA (Fig. 1). The lesion elevated the bony sella causing superior displacement of the pituitary. The lesion extended into left orbital foramina. Mild intracranial extension into the left temporal lobe was also present. 


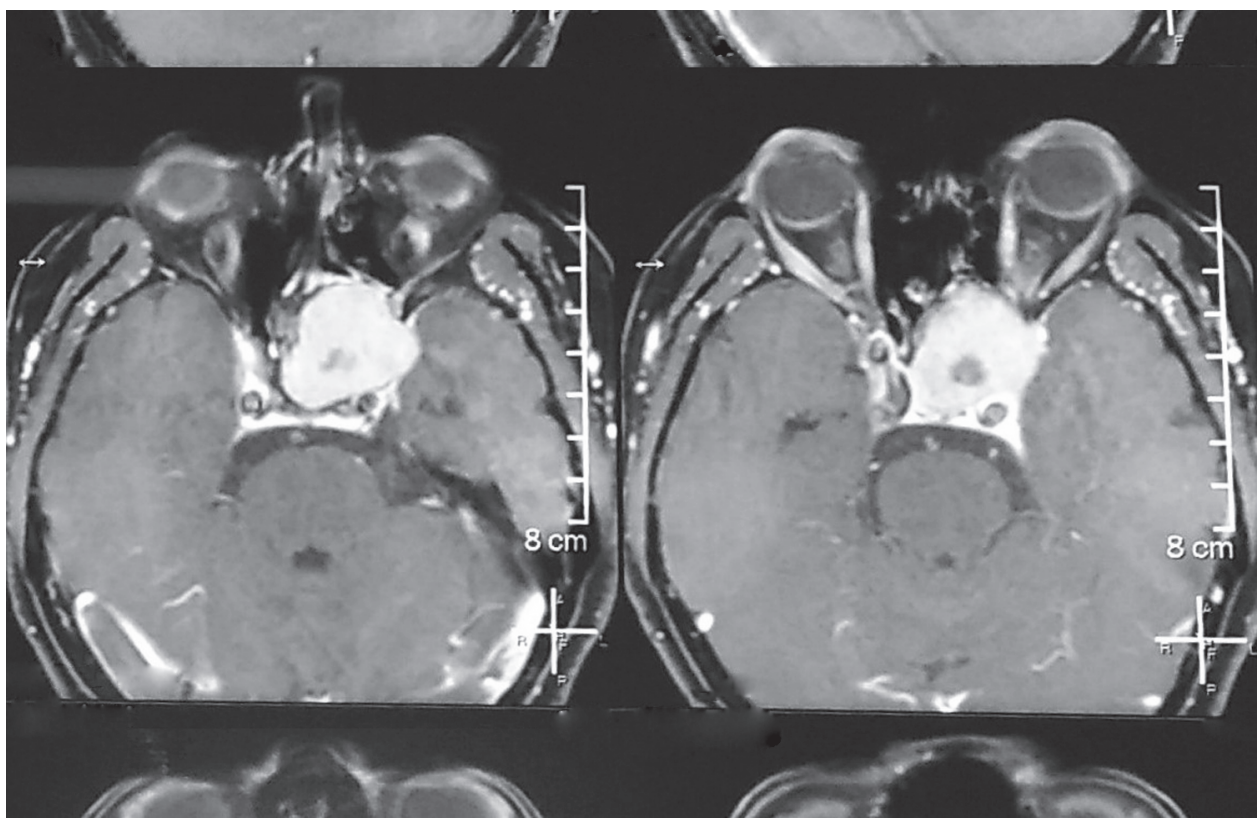

Fig. 1: Preoperative MRI showing an expansile cystic lesion in the left sphenoid sinus

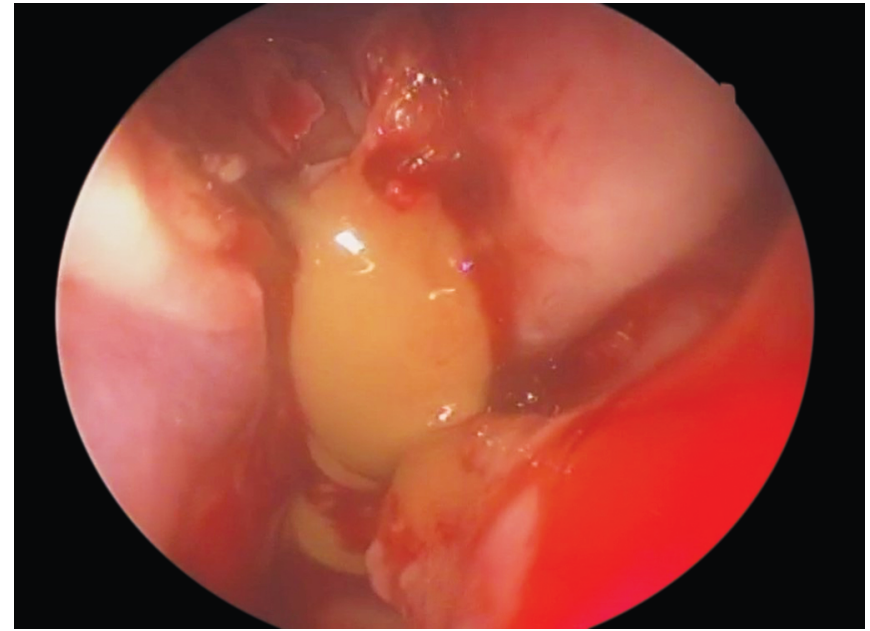

Fig. 2: Drainage of the mucocele

We did drainage of mucocele with marsupialization under GA on Feb 24, 2018. Adequate septal correction, adhesiolysis, and posterior septectomy were also done. After removing rostrum of sphenoid and intersinus septum, the mucocele was marsupialized (Fig. 2). The clival bone was drilled out widening the entire face of sphenoid sinus ensuring adequate drainage. Posterior fossa dura was found exposed. No CSF leak was noted. The cavity was thoroughly cleaned and collected material was sent for investigation. The cavity was packed with surgicel and gelfoam.

The fungal culture was suggestive of aspergillus. Postoperatively, the patient took voriconazole orally for 3 months. Her vision improved to $6 / 9$ on postoperative day 10 , but vision in the temporal region remained as before. She followed up once every month for the next 6 months, during which check nasal endoscopy showed no sign of recurrence and sinus mucosa was healthy each time (Fig. 3). The cavity had healed and completely mucosalized within 3 months. Post-op CT scan done after 6 months showed no sign of

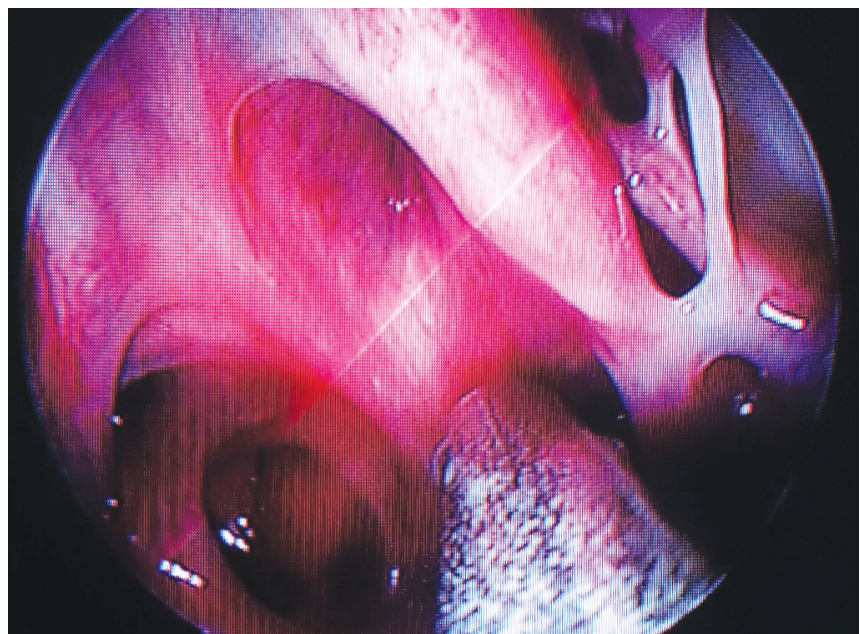

Fig. 3: Nasal endoscopy after 6 months of the surgery

recurrence (Fig. 4). Thereafter, she has been visiting us once every 6 months with no signs of recurrence.

\section{Discussion}

Sinus mucoceles are epithelium-lined cystic masses resulting from obstruction of sinus ostia. Sphenoid sinus mucoceles represent $1-2 \%$ of all paranasal sinus mucocles. ${ }^{1}$ The main clinical features are headache and visual disturbances. Other clinical features depend upon their extension to adjacent structures. Lawson et al. found that the most common symptom is headache. ${ }^{2}$ Soon et al. found ocular symptoms to be the commonest. ${ }^{3}$ Our patient had both visual symptoms and headache. Prolonged pressure on the bone by the mucocele causes erosion of bone and subsequent circulatory changes in the optic nerve. This produces gradual loss of vision. ${ }^{4}$ Heylbroeck et al. reported a case of sphenoidal mucocele, presenting with bilateral visual disturbance along with pituitary gland dysfunction but without nasal or sinus complaints. ${ }^{5}$ 


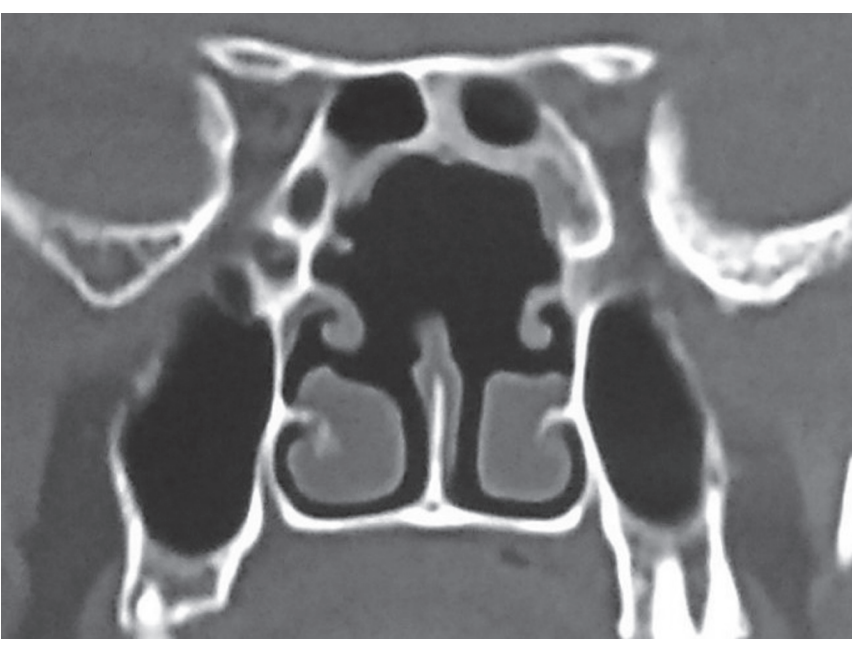

Fig. 4: CT scan after 6 months of surgery showing no signs of recurrence

Radiological imaging should be done for accurate and timely diagnosis. Bone erosion and clival and sella turcica involvement should be specifically looked for. On MRI, the signal intensity of mucocele varies with the presence of a proteinaceous component, hydration, or hemorrhage. They normally show low signal intensity on T1-weighted images and signal void on T2-weighted images due to inspissated debris.

Marsupialization of mucocele via the endoscopic endonasal approach is the treatment of choice. The prognosis is poor in patients who have already lost their eyesight before undergoing surgery. Nagatani et al. reported a case of sphenoid sinus mucocele extending into the middle cranial fossa and the pterygomaxillary fissure, where they used endoscope combined with the navigation system for removal. ${ }^{6}$

\section{Conclusion}

Sphenoid sinus mucoceles are difficult to diagnose and treat because of nondependent drainage, soft tissue, and bony barriers. In cases of recurrent sphenoid sinus mucoceles, complete marsupialization of the mucocele, drilling of bony barriers, adequate medical management, and diligent follow-ups are necessary for successful outcome.

\section{Clinical Significance}

Recurrent mucoceles of the sphenoid sinus represent a difficult entity to treat and are ridden with complications. Not only do they require aggressive surgical management but also meticulous postoperative medical management and repeated endoscopic follow-ups.

\section{References}

1. Kosling $\mathrm{S}$, Hintner M, Brandt $\mathrm{S}$, et al. Mucoceles of the sphenoid sinus. Eur J Radiol 2004;51(1):1-5. DOI: 10.1016/j.ejrad.2003.09.002.

2. Lawson W, Reino AJ. Isolated sphenoid sinus disease: an analysis of 132 cases. Laryngoscope 1997;107(12 Pt 1):1590-1595. DOI: 10.1097/00005537-199712000-00003.

3. Soon SR, Lim CM, Singh H, et al. Sphenoid sinus mucocele: 10 cases and literature review. J Laryngol Otol 2010;124(1):44-47. DOI: 10.1017/ S0022215109991551.

4. Levy J, Monos T, Puterman M. Bilateral consecutive blindness due to SSM with unilateral partial recovery. Can J Ophthalmol 2005;40(4):506-508. DOI: 10.1016/S0008-4182(05)80015-8.

5. Heylbroeck P, Watelet JB, Delbeke $P$, et al. Vision impairment as presenting symptom of a sphenoidal mucocele. Rhinology 2003;41(3):187-191.

6. Nagatani T, Saito K, Yoshida J. Treatment of a sphenoid mucocele using an endoscope combined with a navigating system: a case report. J Clin Neurosci 2001;8(5):456-460. DOI: 10.1054/jocn.2000.0815. 